\title{
Complex stenosis morphology and vasomotor responses to inhibition of nitric oxide synthesis
}

\author{
D Tousoulis, C Tentolouris, T Crake, G Goumas, C Stefanadis, P Toutouzas, G Davies
}

\begin{abstract}
Objective-To assess the relation between coronary vasomotor effects of $\mathrm{N}^{\mathrm{G}}$-monomethyl-Larginine (LNMMA) administration and coronary stenosis morphology, length, and severity in patients with stable angina.

Design-In 28 patients ( 24 male, four female) with coronary artery disease and chronic stable angina, intracoronary normal saline and $4 \mu \mathrm{mol} / \mathrm{min}$ LNMMA were infused for four minutes each, followed by an intracoronary bolus of $250 \mu \mathrm{g}$ glyceryl trinitrate. Coronary stenoses were classified as concentric (smooth), eccentric (smooth), or complicated (irregular). The diameters of these stenoses and their adjacent reference proximal segments were measured by quantitative angiography.

Results-During LNMMA infusion a significantly larger proportion of complicated stenoses than concentric and eccentric stenoses constricted by $\geqslant 5 \%(p<0.01)$ and the magnitude of vasoconstriction was greater in complicated than in concentric and eccentric stenoses $(p<0.05)$. For complicated stenoses the magnitude of constriction (in $\mathrm{mm}$ ) with reference to normal saline was greater than that of the concentric and eccentric stenoses $(p<0.05)$, whereas concentric and eccentric stenoses constricted similarly. Irrespective of the type of morphology, there was a correlation ( $p<0.05)$ between both the severity and the length of stenoses and the magnitude of vasoconstriction to LNMMA. A similar proportion of concentric, eccentric, and complicated stenoses showed $\geqslant 5 \%$ increase in diameter with glyceryl trinitrate, and the magnitude of the response was similar in the three groups.
\end{abstract}

Conclusions-In patients with coronary artery disease, the response to LNMMA is greater when stenosis morphology is complex, indicating greater nitric oxide activity. This provides further evidence that plaques with complex morphology are in an active state.

(Heart 2000;84:529-534)

Keywords: endothelium; nitric oxide; coronary artery disease; stenosis morphology

Nitric oxide is an endogenous vasodilator produced by vascular endothelial cells. ${ }^{1-5}$ Its synthesis can be specifically and competitively antagonised by arginine analogues, such as $\mathrm{N}^{\mathrm{G}}$-monomethyl-L-arginine (LNMMA), which inhibit the enzymatic pathway in a process that can be reversed by increased availability of L-arginine. ${ }^{6-8}$ Inhibition of nitric oxide synthesis has been shown to decrease the basal diameter of angiographically normal and diseased epicardial coronary arteries and stenoses. ${ }^{9-11}$

Evidence suggests that the obstruction asso-

Cardiology Unit, Hippokration Hospital, Athens University Medical School, Greece

D Tousoulis

C Tentolouris

G Goumas

C Stefanadis

P Toutouzas

Cardiology Unit, Imperial College School of Medicine, Hammersmith

Hospital, Du Cane

Road, London W12

ONN, London, UK

T Crake

G Davies

Correspondence to: Dr Davies

g.davies@ic.ac.uk

Accepted 19 July 2000

dynamic rather than fixed. ${ }^{12}{ }^{13}$ A $10 \%$ circumferential shortening of the normal wall may lead to a greater change in luminal calibre at the site of an eccentric plaque than at the site of a concentric plaque. ${ }^{14}{ }^{15}$ Furthermore, coronary stenosis morphology may be an important factor determining the degree of vasomotor response to stimulation. ${ }^{16}{ }^{17}$ Previous studies in animals have shown that the intracoronary activation of platelets and their interaction with the vascular endothelium may contribute to or precipitate myocardial ischaemia, both by causing mechanical obstruction of the arterial lumen and by releasing potent vasoconstrictor substances such as endothelin, thromboxane, or serotonin. ${ }^{18-22}$ Further studies provide evidence of a role for these mechanisms in man. ${ }^{23}{ }^{24}$ Experimental studies ${ }^{25}$ show that the effects of serotonin or acetylcholine differ depending on whether the stenosis is compliant or fixed. A clinical study has shown that the response of stenoses to serotonin is related to the morphology of the lesion. ${ }^{16}$ The relation between nitric oxide activity and coronary stenosis morphology has not been studied.

To study the vasomotor effects of LNMMA administration and their relations to coronary stenosis morphology, we examined the effect of intracoronary LNMMA infusion on coronary luminal diameter, using quantitative angiography, at the site of concentric, eccentric, and complicated lesions in patients with chronic stable angina.

\section{Methods}

PATIENTS

We studied 28 patients (24 male, four female, mean (SD) age 58 (8) years) with chronic stable angina, coronary artery disease, and a positive treadmill exercise test result ( $\geqslant 0.1 \mathrm{mV} \mathrm{ST}$ segment depression) at between 5 and 7 METS using the modified Bruce protocol. All patients were subjected to coronary angiography to assess their suitability for percutaneous transluminal coronary angioplasty (PTCA) or coronary surgery, because their angina limited their physical activity despite drug treatment. Patients were excluded from the study if they had diabetes mellitus, recent myocardial infarction (within six months), left ventricular hypertrophy (on 
echocardiography), left ventricular dysfunction (left ventricular ejection fraction $<50 \%$ ) or valvar heart disease, were current smokers, or had uncontrolled hypertension. Antianginal drug treatment was stopped 24 hours before the study. The patients were allowed to use sublingual glyceryl trinitrate as necessary, but no study was performed within three hours of its administration. The protocol was approved by the research ethics committee and each patient gave written informed consent.

PROTOCOL

Following the diagnostic coronary angiogram an optimal radiographic projection was selected and kept constant for subsequent angiograms (study operators CT, GJD). Thirty eight stenoses and their reference proximal segments were examined. Two ECG leads were monitored continuously throughout the study. Patients received a single four minute infusion of $0.9 \%$ saline $(2 \mathrm{ml} / \mathrm{min})$ followed by a four minute intracoronary infusion of LNMMA ( $4 \mu \mathrm{mol} / \mathrm{min})$ in saline, using a syringe pump, followed after five minutes by an intracoronary bolus dose of glyceryl trinitrate $(250 \mu \mathrm{g}$ in $2 \mathrm{ml}$ of saline). Femoral arterial pressure and heart rate were recorded during the last 30 seconds of each infusion period. Angiography was performed with a hand injection of $6-8 \mathrm{ml}$ non-ionic contrast medium at baseline, immediately after each infusion, and two to three minutes after glyceryl trinitrate. Before each angiogram, the catheter was emptied to avoid bolus administration of the infusate.

QUANTITATIVE CORONARY ANGIOGRAPHY

End diastolic frames from each arteriogram were selected for analysis. Each frame was analysed, in random order, using quantitative computerised analysis with an automated edge contour detection analysis system (Computerised Angiographic Analysis System (CAAS), version 2V2; Pie Data Medical, Maastricht, The Netherlands). ${ }^{16}{ }^{26}$ The angiographic catheter was used as a scaling device and this, together with pincushion distortion correction, allowed the arterial segment diameters to be recorded as absolute values (expressed in millimetres).

Recorded variables at baseline and after saline, LNMMA, and nitrate administration were as follows:

- The luminal diameter of an angiographically normal reference segment (lumen diameter reduction $<20 \%$ ), the distal limit of which was $1-3 \mathrm{~mm}$ proximal to the stenotic site.

- The minimum lumen diameter at the site of coronary stenosis.

- Qualitative and quantitative classification of stenosis morphology.

Stenoses were morphologically classified qualitatively as concentric, eccentric, or "complicated" by two blinded independent observers (DT, CT), on the basis of visual inspection of arteriograms recorded in two orthogonal projections. Ninety five per cent of stenoses were classified in the same way by both observers; the remaining $5 \%$ were classified by consensus. This classification of stenosis mor- phology was compared with that obtained by computed symmetry analysis (CAAS symmetry index $)^{1327}$ of the same coronary lesions. Concentric stenoses were defined as those producing symmetrical narrowing, with smooth borders or only very mild irregularity (symmetry index >0.5-1), that looked similar in orthogonal projections. Eccentric stenoses were defined as asymmetrical narrowing with smooth borders and a broad neck (symmetry index $0.0-\leqslant 0.5$ ). Complicated stenoses were defined as asymmetric narrowing with irregular borders and/or overhanging edges (type II of Ambrose and colleagues ${ }^{29}$ ) or with an "abrupt proximal face" 30 or a "rough" or "sawtooth" component. ${ }^{30}$ Illustrations of these three morphological types of stenosis have been published previously. ${ }^{16}$ Symmetry index was calculated from two parameters, $\mathrm{dl}$ and $\mathrm{dr}$, the smaller divided by the larger, where: $\mathrm{dl}=$ the distance between the detected and estimated left hand contour of the vessel; and $\mathrm{dr}=$ the distance between the detected and estimated right hand contours. The symmetry measure is computed as the minimum of $\mathrm{dl}$ and $\mathrm{dr}$ divided by the maximum of $\mathrm{dl}$ and $\mathrm{dr}$. Accordingly a symmetry index of 1 denotes a concentric stenosis and the value decreases with increasing eccentricity.

Quantitative analysis of coronary arteriograms was carried out by two independent observers (DT, GG), who blindly reanalysed the films at a remote time for reproducibility of the method. No significant intraobserver (analysis of variance $\mathrm{F}=0.4, \mathrm{p}=0.7$ ) or interobserver (analysis of variance $\mathrm{F}=0.35$, $\mathrm{p}=0.82$ ) variability was found. The findings of computed and qualitative assessments of symmetry were concordant in 35 stenoses (92\%) and discordant in three, which were classified by consensus. In a previous study, ${ }^{16}$ we analysed the intraobserver and interobserver variability of the measurement of coronary lumen diameter using the CAAS system in our institution. Twenty four arterial segments were analysed by two independent observers and reanalysed at a remote time. The mean intraobserver variation, expressed as the standard error of the estimate (SEE), was $0.12 \mathrm{~mm}$; the interobserver variation (SEE) was $0.10 \mathrm{~mm}$.

\section{STATISTICAL ANALYSIS}

Data are expressed as mean (SEM). Analysis of variance (ANOVA) and the Scheffé F test for repeated measures were used to compare serial changes in heart rate and blood pressure and in the diameter of coronary stenoses. To test for differences in percentage response to LNMMA and nitrates of concentric, eccentric, and complicated stenoses, a two way ANOVA for repeated measures was applied. Associations between responses to LNMMA and stenosis length, severity, and eccentricity ratio were assessed by performing linear regression analysis and calculating a correlation coefficient. Multivariate analysis was performed between LNMMA response and stenosis length, severity, and eccentricity ratio. Student's $t$ test was used to compare paired and unpaired data 
Table 1 Proportion of stenoses and their reference segments with reactivity ( $\geqslant 5 \%$ change from baseline) to intracoronary $N^{G}$-monomethyl-L-arginine (LNMMA) and nitrates

\begin{tabular}{llllll}
\hline & \multicolumn{2}{l}{ LNMMA } & & & \multicolumn{2}{l}{ Nitrates } \\
\cline { 2 - 3 } \cline { 5 - 6 } \cline { 5 - 6 } & Stenoses & Ref segment & & Stenoses & Ref segment \\
\hline Concentric $(\mathrm{n}=14)$ & $3 / 14(21 \%)$ & $2 / 14(14 \%)$ & & $12 / 14(86 \%)$ & $10 / 14(71 \%)$ \\
Eccentric $(\mathrm{n}=12)$ & $3 / 12(25 \%)$ & $1 / 12(8 \%)$ & & $7 / 12(58 \%)$ & $7 / 12(58 \%)$ \\
Complicated $(\mathrm{n}=12)$ & $8 / 12(67 \%)^{\star}$ & $1 / 12(8 \%)$ & & $10 / 12(83 \%)$ & $10 / 12(83 \%)$ \\
\hline
\end{tabular}

Figures given as $\mathrm{x} / \mathrm{y}(\mathrm{z})$, where $\mathrm{x}=$ number of reactive stenoses or reference segments, $\mathrm{y}=$ total number of stenoses or reference segments, and $z=$ percentage of stenoses or reference segments which are reactive.

${ }^{\star} \mathrm{p}<0.01 v$ concentric and eccentric stenoses.

Ref, reference.

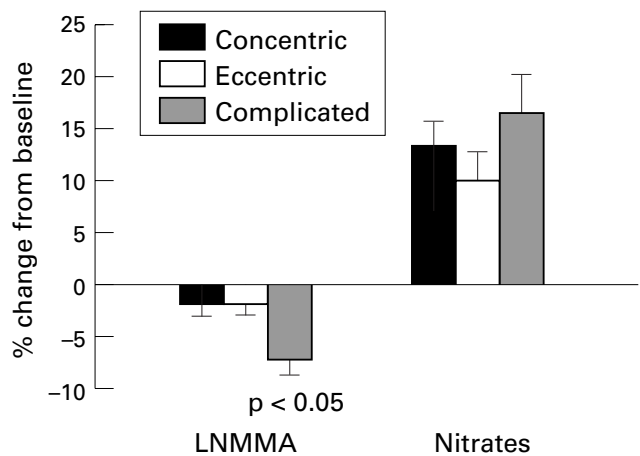

between groups, and the responses to glyceryl trinitrate and to LNMMA. Differences between proportions were analysed by a Yates corrected $\chi^{2}$ test. A probability value of $\mathrm{p}<0.05$ (two tailed) was significant.

\section{Results}

Mean (SEM) systolic aortic pressure increased from 134.0 (3.8) to $143.6 \quad(4.4) \mathrm{mm} \mathrm{Hg}$ $(\mathrm{p}<0.01)$ during intracoronary administration of LNMMA, but heart rate remained unchanged, at 70.0 (1.3) v 70.8 (1.4) beats/ min at baseline. ST segment depression occurred in two patients and was promptly relieved by intracoronary administration of isosorbide dinitrate. Intracoronary glyceryl trinitrate had no significant effect on systolic aortic pressure (134.9 (4.0) v 132.0 (3.6) $\mathrm{mm} \mathrm{Hg}$ at baseline) or heart rate (71.0 (1.2) $v 72.1$ (1.1) beats/min at baseline).

STENOSIS MORPHOLOGY

Thirty eight of the 41 coronary stenoses (14 concentric, 12 eccentric, and 12 complicated) observed in these 28 patients were suitable for quantitative analysis, and the results below refer to these stenoses. Two patients had two stenoses within the same vessel (left anterior descending coronary artery). The severity of coronary stenoses for the whole group ranged from $20.2 \%$ to $91.4 \%$ (mean $46(2.5) \%$ ) lumen diameter reduction. There were 13 stenoses of $\geqslant 50 \%$ : four concentric, three eccentric, and six complicated. There was no significant difference in mean per cent minimum luminal diameter at baseline (44 (3.7)\%, 43 (5.2)\%, and $50.3(4.3) \%$, respectively) or in stenosis length (3.50 (0.30), $3.04(0.31)$, and 3.72 $(0.38) \mathrm{mm}$, respectively) between concentric, eccentric, and complicated stenoses.
Figure 1 Mean coronary stenosis response (per cent change from baseline) to $N^{G}$-monomethyl-L-arginine (LNMMA) and glyceryl trinitrate (nitrates) for concentric, eccentric, and complicated stenoses. In response to LNMMA, complicated stenoses showed significantly greater constriction $(p<0.05)$ than concentric or eccentric stenoses. No differences were found in response to nitrates in these three morphologies.

STENOSIS MORPHOLOGY AND RESPONSE TO LNMMA

During LNMMA infusion a significantly larger proportion of complicated stenoses than concentric and eccentric stenoses constricted by $\geqslant 5 \%(\mathrm{p}<0.01)$, but the response of their proximal reference segments was similar (table 1). The magnitude of vasoconstriction was greater in complicated than in concentric and eccentric stenoses but similar in their proximal reference segments (table 2, figs 1 and 2). Moreover, the magnitude of vasoconstriction was greater than that of the reference segment in complicated stenoses but not in concentric and eccentric stenoses (table 2). For complicated stenoses, the magnitude of constriction (in $\mathrm{mm}$ ) from normal saline was greater than that of the concentric and eccentric stenoses $(p<0.05)$, whereas it was similar for concentric and eccentric stenoses (fig 3). The response to LNMMA of six complicated stenoses with severity $<50 \%$ was similar to six others with severity $\geqslant 50 \%(-9.5(2.3) \%$ and -4.5 (1.7)\%, respectively; NS).

The percentage change in stenosis severity in response to LNMMA was correlated with the severity of stenoses at baseline irrespective of the type of morphology ( $p<0.01$, fig 4 ), and there was a weak correlation $(r=0.39$, $\mathrm{p}<0.05$ ) with the length of stenoses (fig 4). No correlation was found between eccentricity ratio and the response to LNMMA (fig 4). Multivariate analysis showed a correlation

Table 2 Reactivity of coronary stenoses and their reference segments to intracoronary administration of LNMMA and nitrates

\begin{tabular}{|c|c|c|c|c|c|c|}
\hline \multirow[b]{3}{*}{ Morphology } & \multicolumn{6}{|c|}{ Minimum lumen diameter ( $\mathrm{mm}$ ) } \\
\hline & \multicolumn{3}{|l|}{ Stenoses } & \multicolumn{3}{|c|}{ Reference segments } \\
\hline & Baseline & $L N M M A$ & Nitrates & Baseline & $L N M M A$ & Nitrates \\
\hline Concentric $(n=14)$ & $1.38(0.11)$ & $\begin{array}{r}1.36(0.11) \\
-1.9(1.2)^{\star}\end{array}$ & $\begin{array}{r}1.57(0.14) \\
+13.3(2.5)\end{array}$ & $2.50(0.14)$ & $\begin{array}{l}2.42(0.14) \\
-2.8(0.7)\end{array}$ & $\begin{aligned} & 2.71(0.16) \\
+ & 8.7 \quad(1.7)\end{aligned}$ \\
\hline Eccentric $(n=12)$ & $1.64(0.15)$ & $\begin{array}{l}1.62(0.16) \\
-1.8(1.1)^{\star *}\end{array}$ & $\begin{aligned} & 1.80(0.18) \\
+ & 10.1 \quad(2.6)\end{aligned}$ & $2.93(0.13)$ & $\begin{array}{l}2.91(0.14) \\
-0.5(1.4)\end{array}$ & $\begin{aligned} & 3.17(0.14) \\
+ & 8.6(2.2)\end{aligned}$ \\
\hline Complicated $(\mathrm{n}=12)$ & $1.33(0.12)$ & $\begin{array}{l}1.24(0.11) \\
-7.0(1.6)\end{array}$ & $\begin{aligned} & 1.55(0.13) \\
+ & 16.5(3.7)\end{aligned}$ & $2.75(0.17)$ & $\begin{array}{l}2.73(0.18) \\
-1.2(1.1)\end{array}$ & $\begin{array}{r}3.03(0.17) \\
+10.8(2.1)\end{array}$ \\
\hline
\end{tabular}

Values are mean (SD). Values in italics are percentage change from baseline.

${ }^{\star} \mathrm{p}<0.05 ;{ }^{\star \star} \mathrm{p}<0.01 v$ complicated stenoses. 


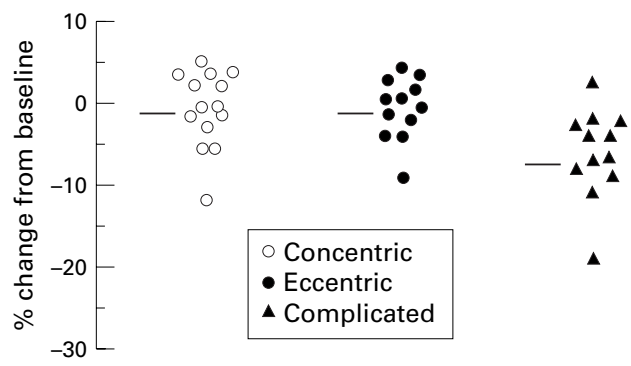

Figure 2 Individual lumen diameter per cent change from baseline in concentric, eccentric, and complicated stenoses after LNMMA infusion. Horizontal bars indicate the mean values.
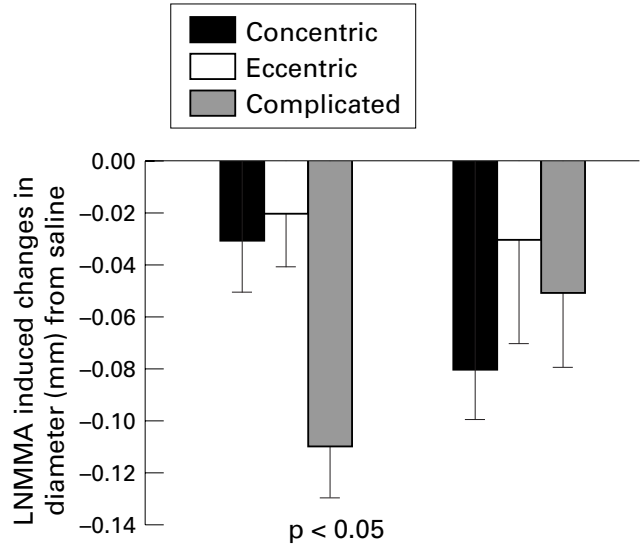

Stenoses Ref segment

Figure 3 The magnitude of stenosis and of reference (ref) segment constriction are expressed as diameter after saline minus diameter after LNMMA (mm) for concentric, eccentric, and complicated stenoses. Complicated stenoses showed significantly greater constriction $(p<0.05)$ than concentric or eccentric stenoses.

between stenosis severity and LNMMA responses as well $(p=0.05)$.

STENOSIS MORPHOLOGY AND RESPONSE TO GLYCERYL TRINITRATE

There was no difference in the effect of glyceryl trinitrate on proximal reference segments of concentric, eccentric, and complicated stenoses (table 2). A similar increase in lumen diameter of concentric, eccentric, and complicated stenoses (table 2, fig 1) occurred, and a similar proportion showed $\geqslant 5 \%$ increase from baseline (table 1).

\section{Discussion}

The results of this study show that complicated (irregular) coronary stenoses are more likely to constrict significantly and constrict to a greater degree in response to LNMMA than eccentric (smooth) and concentric (smooth) stenoses in patients with chronic stable angina. Furthermore, the enhanced constriction is focal at the site of the stenosis, as indicated by the results showing a larger change in lumen diameter at the site of the stenosis than at the adjacent reference arterial segment. They provide evidence to support the hypothesis that complex morphology is characteristic of an active lesion producing nitric oxide. There was also a correlation between the response to LNMMA and stenosis severity and length, but no correlation with eccentricity ratio.

\section{ATHEROMATOUS STENOSES AND NITRIC OXIDE} ACTIVITY

Nitric oxide reduces vascular smooth muscle tone by stimulation of soluble guanylate cyclase $^{7}$ and has an antioxidant effect. ${ }^{31}$ However, when it combines with equimolar amounts of superoxide, peroxynitrite is formed, which is a strong oxidant. Inducible nitric oxide synthase can produce large amounts of nitric oxide and is present in human atherosclerotic lesions. ${ }^{32}$ It has been suggested that diseased arteries may be relatively deficient in the substrate $\mathrm{L}$-arginine. ${ }^{33}$ Apart from limiting nitric oxide production, substrate deficiency could lead to the generation of superoxide by both inducible and endothelial nitric oxide synthase. ${ }^{34}$

The absence of significant response to LNMMA of smooth (concentric and eccentric) stenoses despite a dilator response to nitrates suggests that very little basal production of nitric oxide occurs at this site. This would be consistent with the destruction or malfunction of endothelium which is known to occur at the site of atheromatous plaques. There does appear, however, to be significant variation between patients, as $20-25 \%$ of these stenoses constricted by more than $5 \%$. The correlation of stenosis severity with the response to LNMMA could indicate enhanced nitric oxide production related to greater shear stress at the site of severe stenoses. However, increased severity of stenosis may be related to greater bulk and complexity of the plaque and it is therefore difficult to identify any contribution from stenosis severity as an isolated entity. The response of the six most severe complicated stenoses was not significantly different
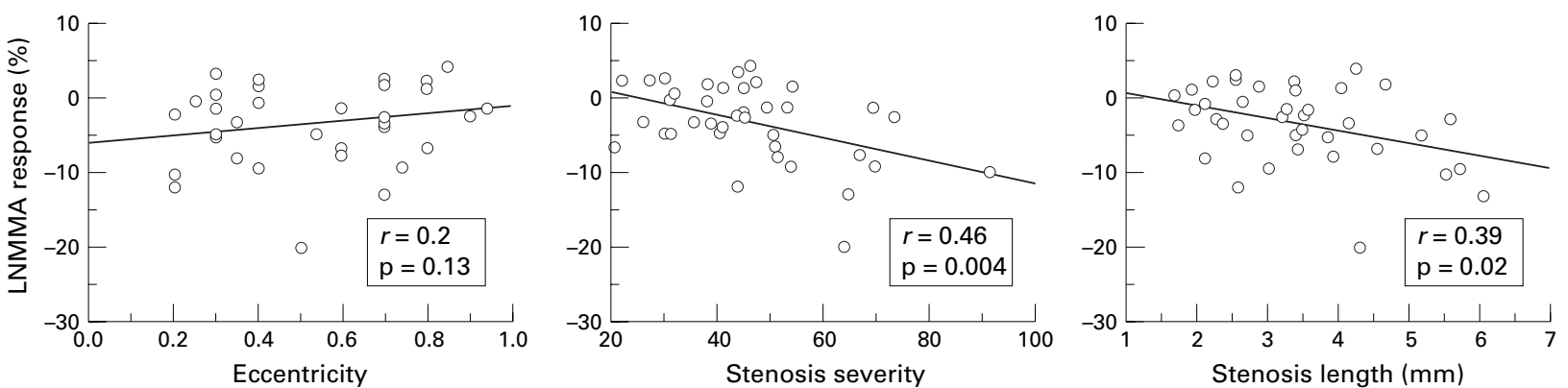

Figure 4 Correlations between stenosis length (right panel), stenosis severity (middle panel), stenosis eccentricity ratio (left panel), and the magnitude of LNMMA response, expressed as per cent change in coronary artery diameter from baseline. Linear regression analysis showed a significant correlation between stenosis severity and LNMMA response, and between stenosis length and LNMMA response. 
from that of the six least severe complicated stenoses.

COMPLEX PLAQUES AND NITRIC OXIDE ACTIVITY Clinical studies have suggested that stenoses with a complex morphology rapidly progress to total or subtotal occlusion and are often the substrate for acute coronary syndromes. ${ }^{28-31} 35$ Our data indicate the presence of enhanced nitric oxide synthase activity (possibly the inducible form) at the site of complex plaques. A possible explanation for this is that nitric oxide production at the site of stenosis occurs in the endothelium of the new microvessels in the wall of the artery around the atheromatous plaque or in other cell types in the vascular wall such as macrophages and smooth muscle cells. Such neovascularisation of atheromatous plaques is well documented and a recent study ${ }^{36}$ showed the presence of inducible nitric oxide synthase in the neomicrovasculature. Nitric oxide production from endothelial cells lining the epicardial segment lumen could be increased because of greater shear stress at the site of complex stenoses. Furthermore, platelet derived nitric oxide activity, as has been shown recently, ${ }^{37}$ could be increased by greater platelet adhesion and aggregation at the site of complex stenoses.

There may be a link between enhanced inducible nitric oxide activity (produced by macrophages) and plaque instability. An abundance of macrophages is often found in such plaques. $^{38-40}$ The macrophages and the $T$ lymphocytes may induce nitric oxide synthase mediated by cytokines. ${ }^{41}{ }^{42}$ Furthermore, metalloproteinases produced in macrophages in vulnerable regions of human atherosclerotic plaques $^{39} 40$ may weaken the fibrous cap of the plaque. There is also growing evidence that macrophages may be involved in smooth muscle cell death by apoptosis occurring in plaques, perhaps reflecting a decline in growth factors or the activity of macrophages. ${ }^{43}$ It is well known that macrophages have the ability to initiate or enhance the degradation of collagen and to promote the propensity of those plaques to rupture and trigger thrombosis. ${ }^{38}$

It might appear that our results indicating enhanced basal nitric oxide activity within complex irregular plaques are in conflict with our previous observation of an enhanced constrictor response of complex stenoses to intracoronary serotonin or acetylcholine. ${ }^{16}{ }^{17}$ However, a constrictor response to serotonin and acetylcholine does not necessarily indicate a reduction in endothelial dilator function as these two agents have a direct constrictor effect on vascular smooth muscle. The constrictor response could reflect smooth muscle cell hyperreactivity, and the coexistence of such hyperreactivity with increased nitric oxide synthase activity cannot be excluded. The results of this study provide further evidence that complex plaques are active structures. Irrespective of increased nitric oxide activity and vascular smooth muscle reactivity, the resting tone appears to be similar for both smooth and complicated stenoses, as evidenced by the similar magnitude of dilatation in response to nitrate administration.

CLINICAL IMPLICATIONS

As the incidence of complex stenosis morphology is about $60 \%$ in patients with unstable angina before myocardial infarction compared with about $20-30 \%$ in patients with chronic stable angina, greater emphasis should be placed on the detection of complex stenosis. ${ }^{29} 44$ This will require the development of new and preferably non-invasive methods of detecting active plaques. An improved understanding of the cellular and molecular mechanism of plaque instability is required.

\section{CONCLUSIONS}

Basal nitric oxide activity in atheromatous plaques is closely related to the morphology of stenosis. It is greater at the site of complicated (irregular) stenoses than at the site of smooth stenoses. This may be related to the cellular composition of complex lesions.

1 Palmer RMJ, Ashton DS, Moncada S. Vascular endothelial cells synthesise nitric oxide from L-arginine. Nature 1988;333:664-6.

2 Palmer RMJ, Ferrige AG, Moncada S. Nitric oxide release accounts for the biological activity of endothelium-derived relaxing factor. Nature 1987;327:524-6.

3 Vanhoutte PM. The endothelium. Modulator of vascular smooth muscle tone. N Engl f Med 1988;319:512-13.

4 Griffith TM, Edwards DH, Lewis MJ, et al. The nature of Griffith TM, Edwards DH, Lewis MJ, et al. The nature of
endothelium-derived relaxing vascular factor. Nature 1984; 308:645-7.

5 Hutchinson PJA, Palmer RMJ, Moncada S. Comparative pharmacology of EDRF and nitric oxide on vascular strips. Eur f Pharmacol 1987;141:445-51.

6 Rees DD, Palmer RMJ, Hodson HF, et al. A specific inhibitor of nitric oxide formation from L-arginine attenuates endothelium-dependent relaxation. Br f Pharmacol 1989; 96:418-24.

7 Woodman OL, Dusting GJ. N-Nitro L-arginine causes coronary vasoconstriction and inhibits endotheliumdependent vasodilatation in anaesthetized greyhounds. $\mathrm{BrF}$ Pharmacol 1991;103:1407-10.

8 Chu A, Chambers DE, Lin C, et al. Nitric oxide modulates epicardial coronary basal vasomotor tone in awake dogs. Am $\mathcal{F}$ Physiol 1990;258:H1250-4.

9 Lefroy DC, Crake T, Uren NG, et al. Effects of inhibition of nitric oxide synthesis on epicardial coronary artery caliber and coronary blood flow in humans. Circulation 1993;88: 43-54.

10 Tousoulis D, Davies GJ, Tentolouris C, et al. Effects of inhibition of nitric oxide synthesis in patients with coronary artery disease and stable angina. Eur Heart $\mathcal{F}$ 1997;18:60813

11 Tousoulis D, Tentolouris C, Crake T, et al. Basal and flowmediated nitric oxide production by atheromatous coronary arteries. F Am Coll Cardiol 1997;29:1256-62.

12 Brown BG, Bolson EL, Dodge HT. Dynamic mechanisms in human coronary stenosis. Circulation 1984;48:797-803.

13 Kaski JC, Tousoulis D, Haider W, et al. Reactivity of eccentric and concentric stenoses in chronic stable angina. $\mathcal{F} \mathrm{Am}$ Coll Cardiol 1991;17:627-33.

14 Saner HE, Gobel FL, Salomonowitz E, et al. The disease-free wall in coronary atherosclerosis: its relation to degree of obstruction. 7 Am Coll Cardiol 1985;6:1096-9.

15 Waller BF. The eccentric coronary atherosclerotic plaque: Waller BF. The eccentric coronary atherosclerotic plaque:
morphologic observations and clinical relevance. Clin Cardiol 1989;12:14-20.

16 Tousoulis D, Davies G, McFadden E, et al. Coronary vasomotor effects of serotonin in patients with angina. Relationship to coronary stenosis morphology. Circulation 1993;88:1518-26.

17 Tousoulis D, Crake T, Kaski JC, et al. Enhanced vasomotor responses of complex stenoses to acetylcholine in patients with chronic stable angina. Am f Cardiol 1995;75:725-8.

18 Ashton JH, Ogletree ML, Michel IM, et al. Cooperative mediation by serotonin S2 and thromboxane A2/ prostaglandin $\mathrm{H} 2$ receptor activation of cyclic flow variations in dogs with severe coronary artery stenoses. Circulation 1987;76:952-9.

19 Berkenboom G, Unger P, Fang ZY, et al. Comparison of the responses to acetylcholine and serotonin on isolated canine and human coronary arteries. Cardiovasc Res 1989;23:7807 .

20 Shimokawa H, Aarhus LL, Vanhoutte P. Porcine coronary arteries with regenerated endothelium have a reduced endothelium-dependent responsiveness to aggregating platelets and serotonin. Circ Res 1987;61:256-70. 
21 Parsons AA, Whalley ET, Fenuik W, et al. 5-HT1-like receptors mediate 5-hydroxytryptamine-induced contraction of human isolated basilar artery. Br F Pharmacol 1989; 96:434-49.

22 Blauw GJ, Bom AH, Van Brummelen P, et al. Effects of 5-hydroxytryptamine on capillary and arteriovenous anastamotic blood flow in the human hand and forearm and in the pig hind leg. F Cardiovasc Pharmacol 1991;17:316-24.

23 McFadden EP, Clarke JG, Davies GJ, et al. Effects of intracoronary serotonin on coronary vessels in patients with stable angina and patients with variant angina. N Engl $\mathcal{F}$ Med 1991;324:649-55.

24 Connor HE, Fenuik W, Humphrey PPA. 5-Hydroxytryptamine contracts human coronary arteries predominantly via 5-HT2 receptor activation. Eur $\mathcal{F}$ Pharmacol 1989;161:91-4.

25 Ichikawa $\mathrm{Y}$, Yokoyama M, Akita $\mathrm{H}$, et al. Constriction of a large coronary artery contributes to serotonin-induced myocardial ischemia in the dog with pliable coronary myocardial ischemia in the dog with plabl

26 Reiber JHC, Serruys PW, Koouman C, et al. Assessment of short-, medium, and long-term variations in arterial dimensions from computer-assisted quantitation of coronary cineangiograms. Circulation 1985;71:280-8.

27 Tousoulis D, Kaski JC, Davies G, et al. Preangioplasty complicated coronary stenosis morphology as a predictor to restenosis. Am Heart f 1992;123:15-20.

28 Ambrose JA, Winters SL, Arora RR, et al. Coronary angiographic morphology in myocardial infarction: a link between the pathogenesis of unstable angina and myocardial infarction. F Am Coll Cardiol 1985;6:1233-8.

29 Ambrose JA, Winters SL, Arora RR, et al. Angiographic evolution of coronary artery morphology in unstable angina. 7 Am Coll Cardiol 1986; 7:472-8.

30 Ellis S, Alderman EL, Cain K, et al. Morphology of left anterior descending coronary territory lesions as a predictor of anterior myocardial infarction: a registry study $f$ Am Coll Cardiol 1989;13:1481-91.

31 Ohara Y, Peterson TE, Sayegh HS, et al. Dietary correction of hypercholesterolemia in the rabbit normalises endothelial superoxide anion production. Circulation 1995;92:898903.
32 Mugge A, Brandes RP, Boger FH, et al. Vascular release of superoxide radicals is enhanced in hypercholesterolemic
rabbits. 7 Cardiovasc Pharmacol 1994;24:994-8.

33 Tousoulis D, Davies G, Tentolouris C, et al. Coronary stenosis dilation induced by L-arginine. Lancet 1997;349: 1812-13

34 Rubanyi GM, Vanhoutte PM. Superoxide anions and hyperoxia inactivate endothelium-derived relaxing factor. Am F Physiol 1986;250:H822-7.

35 Tousoulis D, Davies G, Crake T, et al. Angiographic characteristics of the infarct and non-infarct related stenoses in patients with stable angina progressed to acute myocardial infarction. Am Heart f 1998;136:382-8.

36 Buttery LDK, Springall DR, Chester AH, et al. Inducible NO synthase is present within human atherosclerotic lesions and promotes the formation and activity of peroxynitrite. Lab Invest 1996;75:77-85.

37 Freedman JE, Ting B, Hankin B, et al. Impaired platelet production of nitric oxide predicts presence of acute production of nitric Oxide predicts presence

38 Libby P, Geng YJ, Aikawa M, et al. Macrophages and atherosclerosis plaque stability. Curr Opin Lipidol 1996;7: athero

39 Galis ZS, Sukhova GK, Lark MW, et al. Increased expression of matrix metalloproteinases and matrix degrading activity in vulnerable regions of human atherosclerotic plaques. $\mathcal{F}$ Clin Invest 1994;94:2493-503.

40 Dollery CM, McEwan JR, Henney AM. Matrix metalloproteinases and cardiovascular disease. Circ Res 1995;77:8638.

41 Esaki T, Hayashi T, Muto E, et al. Expression of inducible nitric iodide synthase in $\mathrm{T}$ lymphocytes and macrophages of cholesterol-fed rabbits. Atherosclerosis 1997;128:39-46.

42 Schonbeck U, Mach F, Sukhova GK, et al. Regulation of matrix metalloproteinase expression in human vascular smooth muscle cells by T-lymphocytes. Circ Res 1997;81: $448-54$.

43 Saraste A, Pulkki K, Kallajoki M, et al. Apoptosis in human acute myocardial infarction. Circulation 1997;95:320-23.

44 Kaski JC, Chen L, Chester MR. Rapid angiographic progression of coronary artery disease in patients with angina pectoris. Circulation 1995;92:2058-65.

\section{IMAGES IN CARDIOLOGY}

\section{Mitral valve aneurysm}

A 65 year old man with a five month history of low grade fever, fatigue, and asthenia was admitted to our hospital. Physical examination findings were normal except for a grade III/VI systolic murmur at the apex. There was

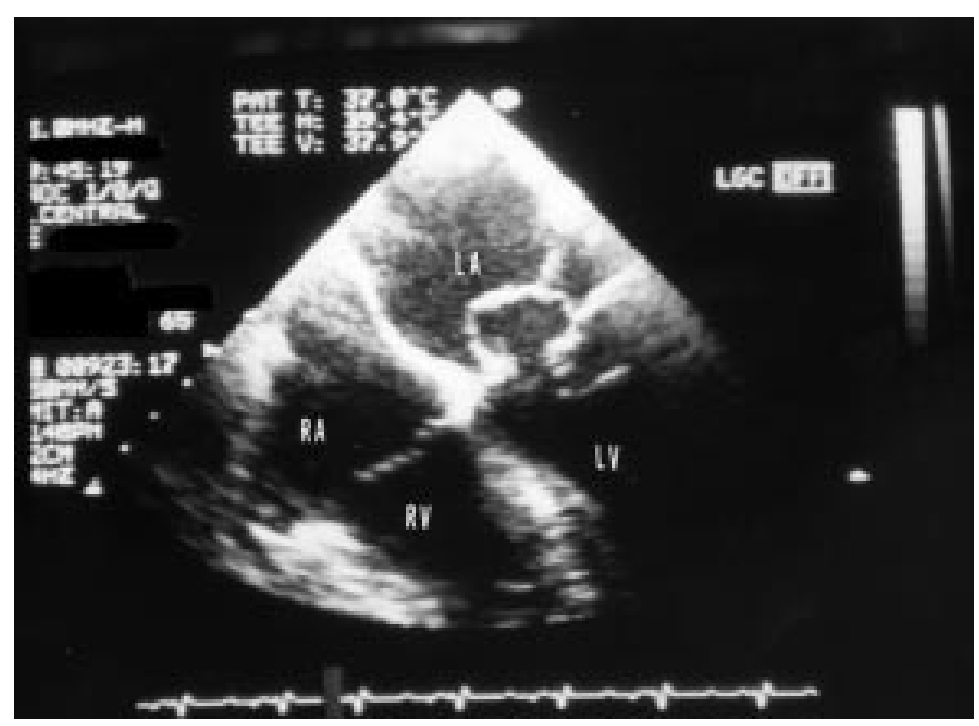

anaemia $(6.3 \mathrm{mmol} / \mathrm{l})$ with an elevated erythrocyte sedimentation rate $(54 \mathrm{~mm} /$ hour$)$. Blood cultures grew Enterococcus faecalis, and the patient was treated with penicillin and gentamicin for six weeks. Transthoracic and transoesophageal echocardiography demonstrated a vegetative mass in the aortic valve, a moderate aortic regurgitation, perforation of the anterior mitral leaflet, and a mitral valve aneurysm (LA, left atrium; LV, left ventricle; RA, right atrium; RV, right ventricle). Doppler examination revealed a moderate to severe mitral regurgitation across the mitral valve aneurysm. Because of mitral and aortic regurgitation and haemodynamic deterioration, the patient underwent aortic and mitral valve replacement. The aneurysm of the anterior mitral valve leaflet was confirmed during surgery. Histologic examination of the resected segments showed focal fibrin deposits and acute inflammation foci, consistent with the diagnosis of infective endocarditis. The postoperative course was uneventful and subsequent blood cultures were negative. 